Research Paper

\title{
Transcatheter arterial chemoembolization (TACE) versus hepatectomy in hepatocellular carcinoma with macrovascular invasion: a meta-analysis of 1683 patients
}

\author{
Jinbin Chen"1,2*, Jia Huang3*, Minshan Chen¹,2, Keli Yang1,2, Jiancong Chen, 2, Juncheng Wang1, 2,Li Xu1,2,

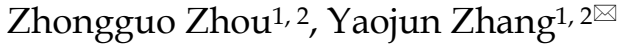 \\ 1. Department of Hepatobiliary Surgery, Sun Yat-sen University Cancer Center, 651 Dongfeng Road East, Guangzhou 510060, China; \\ 2. Sun Yat-sen University Cancer Center, State Key Laboratory of Oncology in South China, Collaborative Innovation Center for Cancer Medicine, \\ Guangzhou 510060, China; \\ 3. Department of Obstetrics and Gynaecology, First Affiliated Hospital of Sun Yat-sen University, 58 Zhongshan Road Second, Guangzhou, 510089, P. R. \\ China. \\ * These two authors contributed to this work equally
}

$\square$ Corresponding author: Yaojun Zhang, M.D. Ph.D., Department of Hepatobiliary Surgery, Sun Yat-sen University Cancer Center, 651 Dongfeng Road East, Guangzhou 510060, China. Telephone: (8620) 8734 3115; Fax: (8620) 8734 3585; Email: zhangyuj@sysucc.org.cn

(c) Ivyspring International Publisher. This is an open access article distributed under the terms of the Creative Commons Attribution (CC BY-NC) license (https:// creativecommons.org/licenses/by-nc/4.0/). See http://ivyspring.com/terms for full terms and conditions.

Received: 2017.05.11; Accepted: 2017.07.03; Published: 2017.08.25

\begin{abstract}
For hepatocellular carcinoma (HCC) patients with macrovascular invasion (MaVI), hepatectomy and transcatheter arterial chemoembolization (TACE) remain the main treatment options in Asia. However, which could achieve better survivals remains controversial. In present study, we conducted a meta-analysis to clarify the survival benefits and safety of hepatectomy versus TACE in HCC patients with MaVI. The PubMed, Cochrane Library and Web of Science databases were searched for eligible studies. There were no prospective studies identified. 8 retrospective studies from Asia with 1683 patients met our inclusion criteria were included in meta-analysis. The overall survival (OS) is higher in hepatectomy group than TACE group $(\mathrm{HR}=1.61,95 \% \mathrm{Cl}=1.23-2.10, p=0.0005)$. Hepatectomy was superior over TACE in 1-year $(O R=2.27,95 \% C l=1.26-4.08, p=0.006)$ and 3-year $(O R=3.04$, $95 \% \mathrm{Cl}=2.17-4.26, p<0.00001)$ respectively, but not in 5 -year $(\mathrm{OR}=7.34,95 \% \mathrm{Cl}=0.78-68.16, p=0.08)$ survival rate. Subgroup analysis demonstrated that hepatectomy was superior over TACE for patients with PVTT $(\mathrm{HR}=1.50,95 \% \mathrm{Cl}=1.14-1.98, p=0.004)$, but not for patients with HVTT/IVC $(\mathrm{HR}=2.39$, $95 \% \mathrm{Cl}=0.88-6.49, p=0.09)$. There was not significantly difference between two groups in peri-operative mortality. Our results indicated that, compared to TACE, hepatectomy might be a better treatment option for resectable HCC patients with MaVI. Being lack of high-quality studies, more well-designed multi-center randomized trials are needed to confirm our finding.
\end{abstract}

Key words: Hepatocellular carcinoma, Marcovascular invasion, hepatectomy, Transcatheter arterial chemoembolization, Meta-analysis.

\section{Introduction}

Hepatocellular carcinoma (HCC) is the fifth most common malignant tumor and the third frequent cause of cancer death worldwide [1]. Hepatectomy is regarded as the first-line therapy for patients with HCC. Unfortunately, only $10 \%-30 \%$ of HCC patients are amenable to such a "curative" therapy when first diagnosed because of multifocality, macrovascular invasion (MaVI) or extra-hepatic metastases. Instead, transcatheter arterial chemoembolization (TACE) could be palliative treatment for unresectable HCC [2, 3].

According to the Barcelona Clinic Liver Cancer (BCLC) treatment guideline approved by European Association for Study of the Liver (EASL), hepatectomy should be recommended option for the BCLC stage 0 or A HCC with single tumor, and TACE 
should be recommended option for BCLC B HCC with multiple tumors. Once MaVI diagnosed, sorafenib is the recommended option. It had been reported that the median survival time for HCC patients with MaVI is less than 4 months if left untreated [4-6], and the median survival time would be extended to about 6-8 months with sorafenib treatment [7-9]. On the contrast, hepatectomy and TACE are still the main treatment options for HCC patients with MaVI in Asia. The investigators from Asia believed that those patients would benefit from more aggressive treatments $[3,6,10]$. The differences in etiology and biological behavior between the East and the West HCC contribute to the divergence in treatment options.

Tumor thrombosis in portal vein will increase portal venous pressure and decrease the blood flow to the liver, which will lead to fatal upper gastrointestinal hemorrhage or liver failure. Remove the tumor thrombosis by surgical treatment might prevent these complications, but the recurrence rate after surgery is relatively high and the median overall survival were reported to be 8.2-27.6 months [6, 11]. Clinical trials and meta-analyses had verified the effectiveness of TACE in prolonging survival in unresectable HCC. For patients with MaVI, the median overall survival after TACE were reported to be 6.1-8.6months $[12,13]$.Although there were some studies comparing the effectiveness of hepatectomy with TACE for HCC patients with MaVI, the results remained controversial. Therefore, we conducted a meta-analysis of available literatures to clarify the survival benefits and safety of hepatectomy over TACE in HCC patients with MaVI.

\section{Methods}

\section{Search strategy}

The PubMed, Cochrane Library and Web of Science databases were searched. Since MaVI can be described as portal/hepatic vein tumor thrombus (PVTT/HVTT), portal/hepatic vein tumor thrombosis, portal/hepatic vein thrombosis or main vein thrombi, we simplified them as "tumor thromb", "vein thromb*" and "vascular invas" (the symbol * means the suffix was simplified). Finally, search items in [Tittle/Abstract] were as follows: (HCC or "hepatocellular carcinoma") AND ("vein thromb" or "tumor thromb" or "vascular invas") AND (hepatectomy or resection or surgery) AND (TACE or "transcatheter arterial chemoembolization" or "transarterial chemoembolization"). The last search was performed on June 6, 2016. Relevant literatures were also manually searched.

\section{Inclusion and exclusion criteria}

All the studies met the following criteria were included in this meta-analysis:1) The study subjects were HCC patients with MaVI;2) Patients received hepatectomy or TACE as initial treatment;3) The studies were prospective RCTs, observational or retrospective observational studies comparing hepatectomy with TACE;4) Data contains outcomes of survivals.

Studies were excluded if they met any of the following criteria: 1) The study subjects were not HCC, for example hepatic metastases;2)Not comparing hepatectomy with TACE;3) TACE before or after hepatectomy;4) Studies based on overlapping cohorts from the same institutions;5) Survival data unavailable; 6)Cases reports or conference reports;

\section{Data extraction and quality assessment}

Two investigators (JBC and JH) independently reviewed all potentially eligible studies. Any disagreement was resolved by adjudicating senior authors (YJZ). The first author, published year, enrollment period, design of the study, inclusion criteria, number of HCC cases in hepatectomy and TACE groups, the survival outcomes were extracted. The primary outcome was the hazard ratios (HRs) comparing hepatectomy with TACE in overall survival (OS) or the Kaplan-Meier curve with log-rand test. The secondary outcomes were 1-year, 3-year and 5-year survival rates. When treatment-related mortality or complication was reported, relevant data was extracted too. If outcome data were incomplete, we asked the contact author for additional information.

The methodological quality of RCTs was assessed on the Cochrane risk of bias tool. The sequence generation, incomplete outcome data, allocation concealment, selective outcome reporting, blinding and other sources of bias will be assessed. The Newcastle-Ottawa Scale(NOS) was used to assess the study quality of the retrospective studies.The NOS uses two different tools for case-control and cohort studies and consists of three parameters of quality: selection(0-4 points), comparability (0-2 points), and outcome assessment (0-3 points). The maximum possible score is 9 points, representing the highest methodological study. And a study with a rating of 7 or more was considered to be of high quality.

\section{Statistical analysis}

All the meta-analyses were performed using Review Manager 5.3(Cochrane Collaboration, Oxford, UK). HRs for OS was not reported in every study. Therefore, we calculated $\ln [\mathrm{HR}]$ with standard error(SE) by using the calculation sheets which were 
developed by Matthew Sydes and Jayne Tierney [14]. Then the $\ln [\mathrm{HR}] \mathrm{s}$ and SEs were pooled according to inverse variance. And the 1-year, 3-year and 5-year survival rates were compared between hepatectomy group and TACE group to get more detail. The odd ratios (ORs) with 95\% CIs were pooled to compare dichotomous variables.

The significance of heterogeneity was assessed using the chi-square test for $p$ values less than 0.1. And the extent of inconsistency was assessed by $I^{2}$ statistic $\left(I^{2}>50 \%\right.$ was considered as having substantial heterogeneity). If the studies did not significantly differ, the fixed-effects model was used. Otherwise, the random-effects did.

Subgroup analyses were performed to compare hepatectomy with TACE in different types of MaVI (PVTT or HVTT/IVC). Sensitivity analyses were performed for high-quality studies. Funnel plots were used to screen for potential publication bias when the number of including studies is larger than 10 according to the cochrane handbook.

\section{Results}

\section{Characteristics of included studies}

A total of 567 publications were identified using the predetermined search strategy. 51 of them were retrieved for detailed evaluation after scanning the title and abstract. All of these 51 publications were read full text and 9 of them met all the criteria [6, 15-22]. But for the studies of Po-Hong Liu and Yun-Hsuan Lee $[17,18]$, the data were from the same hospital in the same period. Because of the lower quality of comparability, the study of Yun-Hsuan Lee was excluded. Finally, 8 publications were included in meta-analysis (Figure 1). The characteristics of the eligible studies are shown in Table 1.

There were no prospective studies with all retrospective studies which published between 2005-2015, and all studies were from Asia including 1 from Korean and 7 from China. Among the studies, 3 were designed as case-matched $[15,17,21]$ and $5[6$, $16,18-20,22]$ were cohort studies. Two retrospective studies declared prospective data collection [19, 21]. 6 studies focused on the patients with PVTT [6, 16-18, 20-22] and 2 focused on HVTT or tumor thrombosis in inferior vena cave (IVC) and right atrium (RA) [15, 19]. According to different vein system, the former was allocated to the PVTT subgroup, and the later was allocated to the HVTT subgroup. The data of 1683 patients, 853 received hepatectomy and 830 received TACE, was extracted.

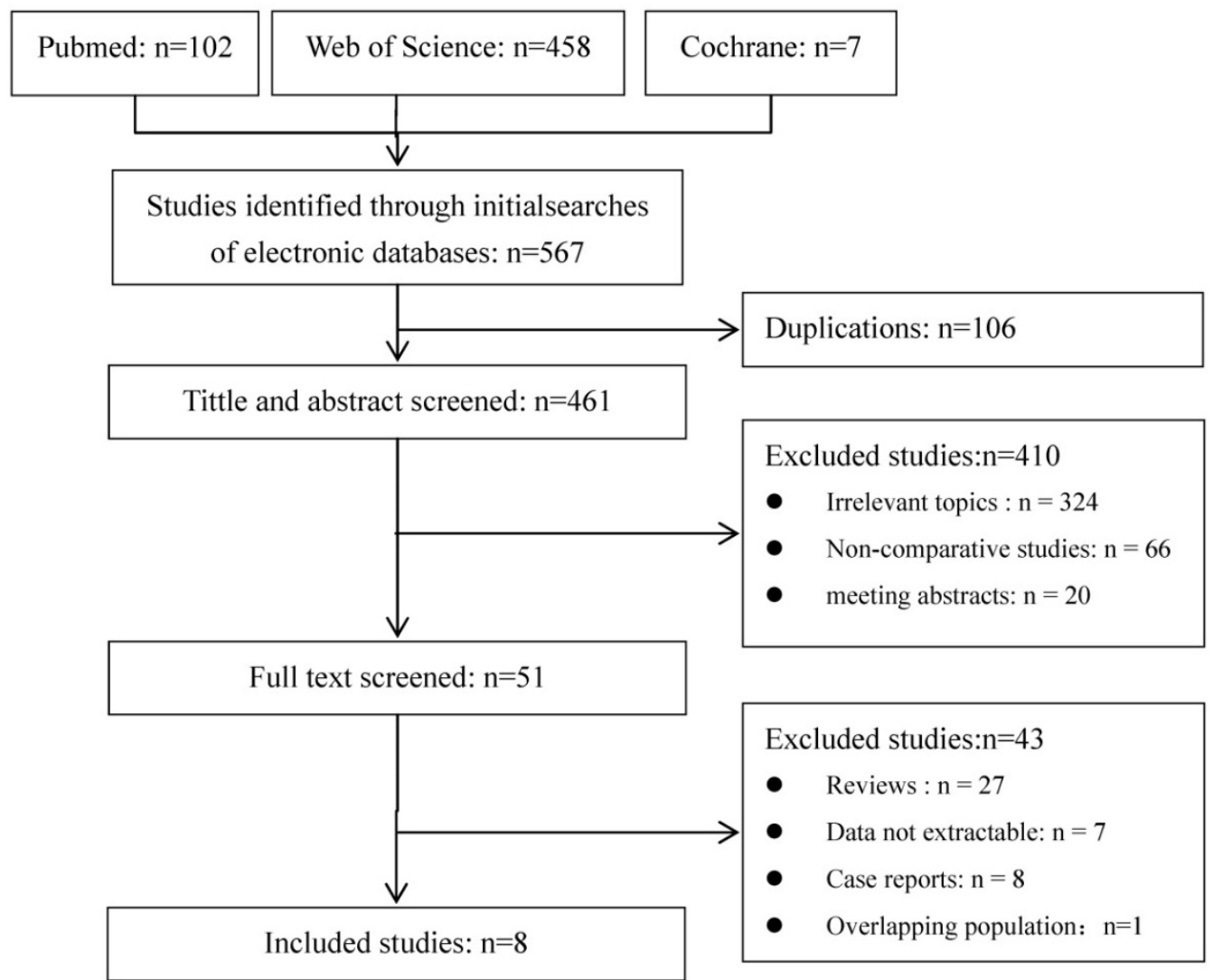

Figure 1. Flow diagram of studies identified, included, and excluded. 
The quality of the studies was assessed by the NOS as pre-mentioned. Methods for handling missing data and intention-to-treat analyses were not adequately described in the majority of studies. 5 studies can be considered as high-quality (Table 2). They are high-quality in comparability. Having a balanced baseline characteristic, influence of important predicting factors between groups were reduced.

Table 1. Characteristics of included studies.

\begin{tabular}{|c|c|c|c|c|c|c|c|c|}
\hline \multirow[t]{2}{*}{ Study } & \multirow{2}{*}{$\begin{array}{l}\text { Published } \\
\text { Year }\end{array}$} & \multirow[t]{2}{*}{ Design } & \multirow{2}{*}{$\begin{array}{l}\text { Type of } \\
\text { vascular } \\
\text { invasion }\end{array}$} & \multicolumn{3}{|l|}{ Case } & \multicolumn{2}{|c|}{ Median Survival time } \\
\hline & & & & Total & Hepatectomy & TACE & $\begin{array}{l}\text { Hepatectomy } \\
\text { (months) }\end{array}$ & $\begin{array}{l}\text { TACE } \\
\text { (months) }\end{array}$ \\
\hline Po-Hong Liu & 2014 & Case-matched & PVTT & 216 & 108 & 108 & 64 & 32 \\
\hline Jia-Ye Zhou & 2014 & Cohort Study & PVTT & 176 & 90 & 86 & 8.2 & 7 \\
\hline Qi Zhou & 2011 & Cohort Study & PVTT & 48 & 38 & 10 & 10 & 7 \\
\hline Yong-Fa Zhang & 2015 & Case-matched & HVTT & 84 & 28 & 56 & 15.6 & 9.1 \\
\hline Zhen-Wei Peng & 2012 & Case-matched & PVTT & 603 & 201 & 402 & $20.0 \pm 1.8$ & $13.1 \pm 0.6$ \\
\hline Yi Wang & 2013 & Cohort Study & IVC/RA & 45 & 25 & 20 & 19 & 4.5 \\
\hline Shu-Qun Cheng & 2005 & Cohort Study & PVTT & 45 & 7 & 38 & 8.0 & 5.0 \\
\hline Liang Ma & 2013 & Cohort Study & PVTT & 176 & 90 & 86 & 8.2 & 7.0 \\
\hline Zhi-Ming Wang & 2015 & Cohort Study & PVTT & 335 & 273 & 62 & 4.65 & 5.65 \\
\hline
\end{tabular}

PVTT: Portal vein tumor thrombosis; HVTT: Hepatic vein tumor thrombosis; IVC: inferior vena cave; AR: right atrium.

Table 2. Results of study quality assessment with NOS

\begin{tabular}{|c|c|c|c|c|c|c|c|c|c|}
\hline \multirow[t]{2}{*}{ Study } & \multicolumn{4}{|l|}{ Selection } & \multirow[t]{2}{*}{ Comparability } & \multicolumn{3}{|l|}{ Outcome } & \multirow[t]{2}{*}{ Scores } \\
\hline & $\begin{array}{l}\text { Representative } \\
\text { treatment group }\end{array}$ & $\begin{array}{l}\text { Representative } \\
\text { reference group }\end{array}$ & $\begin{array}{l}\text { Ascertainment } \\
\text { of exposure }\end{array}$ & $\begin{array}{l}\text { outcome at } \\
\text { start of study }\end{array}$ & & $\begin{array}{l}\text { Assessment } \\
\text { of outcome }\end{array}$ & $\begin{array}{l}\text { Follow-up long } \\
\text { enough for outcomes }\end{array}$ & $\begin{array}{l}\text { Adequate } \\
\text { followup }\end{array}$ & \\
\hline Po-Hong Liu & $※$ & $※$ & - & $※$ & $※ ※$ & $※$ & $※$ & - & 7 \\
\hline Jia-Ye Zhou & $※$ & $※$ & - & $※$ & $※ ※$ & $※$ & $※$ & - & 7 \\
\hline Qi Zhou & $※$ & $※$ & - & $※$ & - & $※$ & - & - & 4 \\
\hline Yong-Fa Zhang & $※$ & $※$ & - & $※$ & $※ ※$ & $※$ & $※$ & - & 7 \\
\hline Zhen-Wei Peng & $※$ & $※$ & - & $※$ & $※$ & $※$ & $※$ & - & 6 \\
\hline Yi Wang & $※$ & $※$ & - & $※$ & $※ ※$ & $※$ & $※$ & - & 7 \\
\hline Liang Ma & $※$ & $※$ & - & $※$ & $※ ※$ & $※$ & $※$ & - & 7 \\
\hline Zhi-Ming Wang & $※$ & $※$ & - & $※$ & - & $※$ & - & - & 4 \\
\hline
\end{tabular}

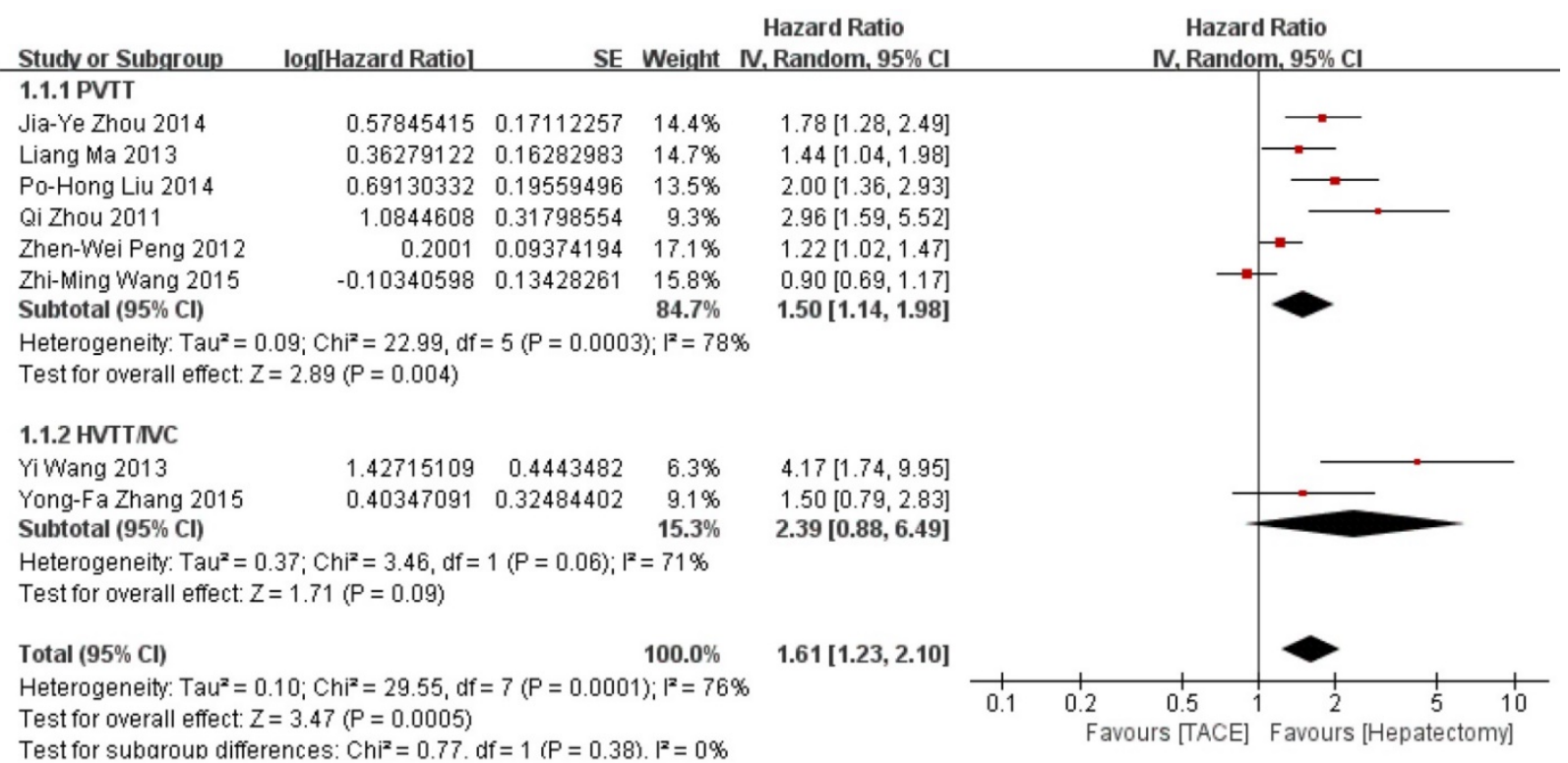

Figure 2. Forest plot and meta-analysis of over survival. Squares are the point estimates of the HRs with the $95 \% \mathrm{Cls}$ indicated by horizontal bars. Diamonds are the summary estimates and $95 \% \mathrm{Cls}$ from the pooled studies. PVTT: Portal vein tumor thrombosis; HVTT: Hepatic vein tumor thrombosis; IVC: tumor thrombosis in inferior vena cave. 
Data on 1-year survival rate were available in all studies. The result of meta-analysis favors hepatectomy with a HR of 2.27 (95\% CI=1.26-4.08, $p=0.006)$. The heterogeneity is significant $(p<0.0001$, $I^{2}=77 \%$, Figure 3). Similarly, the 3 - year survival rate was statistically lower in TACE group $(\mathrm{HR}=3.04$, $95 \% \mathrm{CI}=2.17-4.26, p<0.00001$, Figure 4 ), with 7 studies included and no obvious heterogeneity $(p=0.13$, $\left.I^{2}=39 \%\right)$. Only 2 studies reported the 5 -year survival rate. The meta-analysis demonstrated no significant difference between hepatectomy and TACE (HR=7.34, $95 \% \mathrm{CI}=0.79-68.16, p=0.08)$, although both of the original studies showed a significant difference (Figure 5). The peri-operative mortality was reported in three studies. The meta-analysis demonstrated no significant difference ( $p=0.22$, Figure 6 ).

In the terms which heterogeneity is significant, high-quality studies were assessed, except the 5-year survival rate because of the lack of enough studies. There is no change in terms of the significance of each of the outcomes. But between-study heterogeneity was significantly reduced by the sensitivity analysis for OS, and 1-year survival rate (Table 3). And according to the cochrane handbook, funnel plots were not necessary in our study.

\section{Discussion}

This meta-analysis of 8 retrospective studies comparing hepatectomy with TACE demonstrated that hepatectomy was potentially more effective for HCC patients with MaVI. The overall survival, 1- year and 3- year survival rate were higher in hepatectomy group than in TACE group. At the same time, the treatment-related mortality did not significantly differ between two groups.

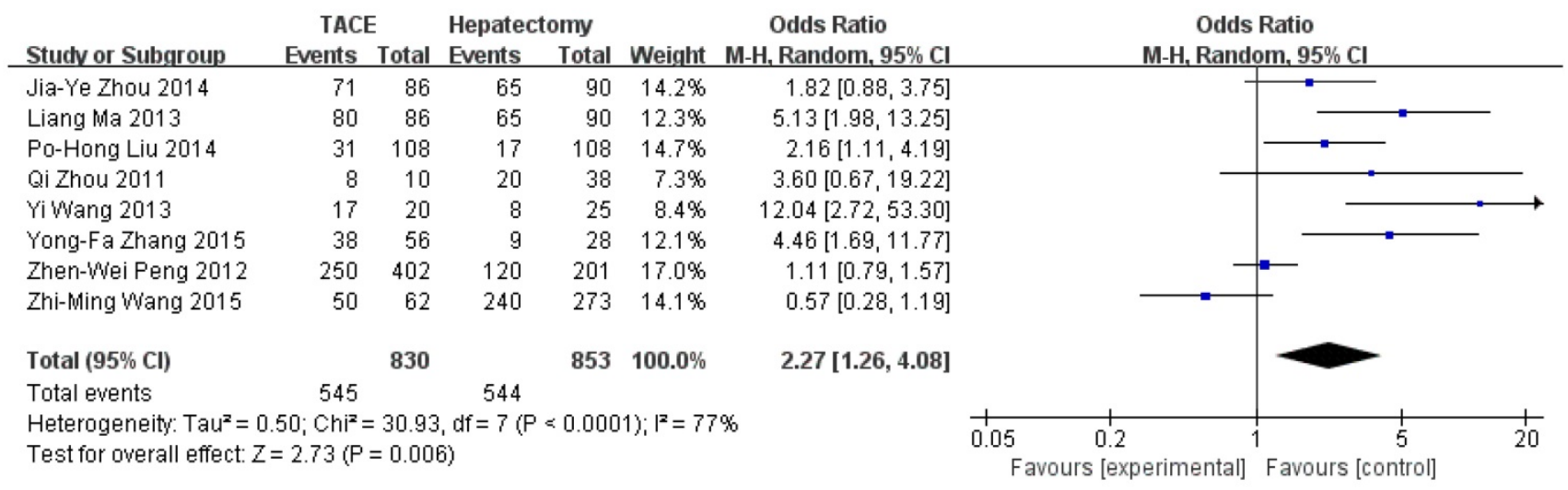

Figure 3. Forest plot and meta-analysis of 1-year Survival Rate.

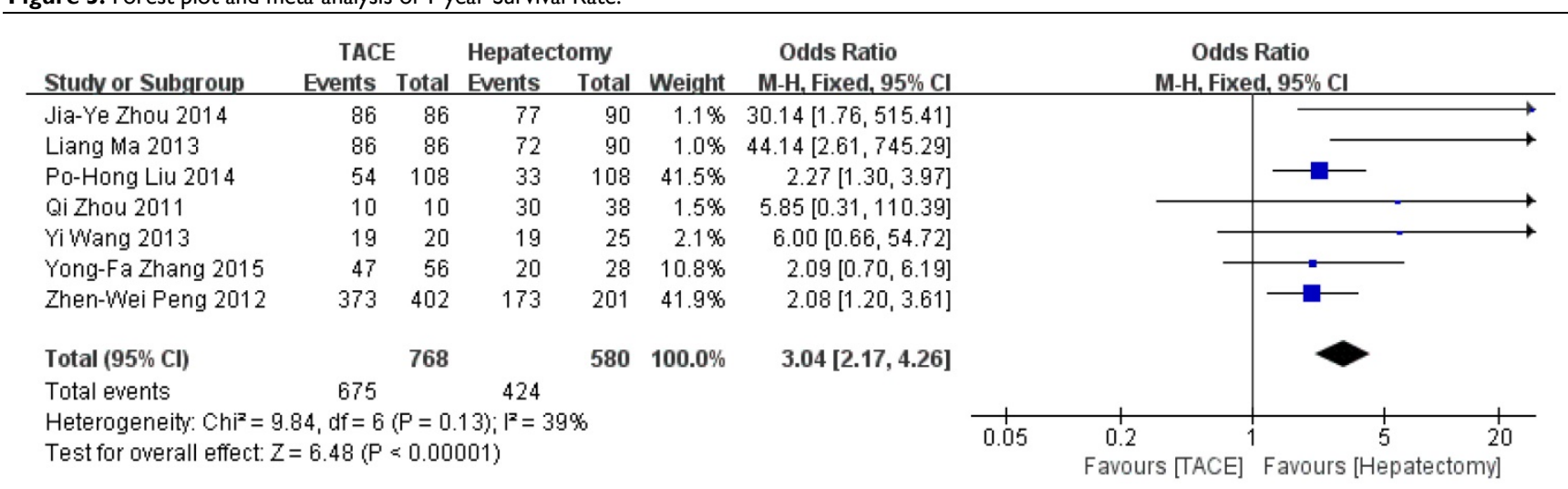

Figure 4. Forest plot and meta-analysis of 3-year Survival Rate.

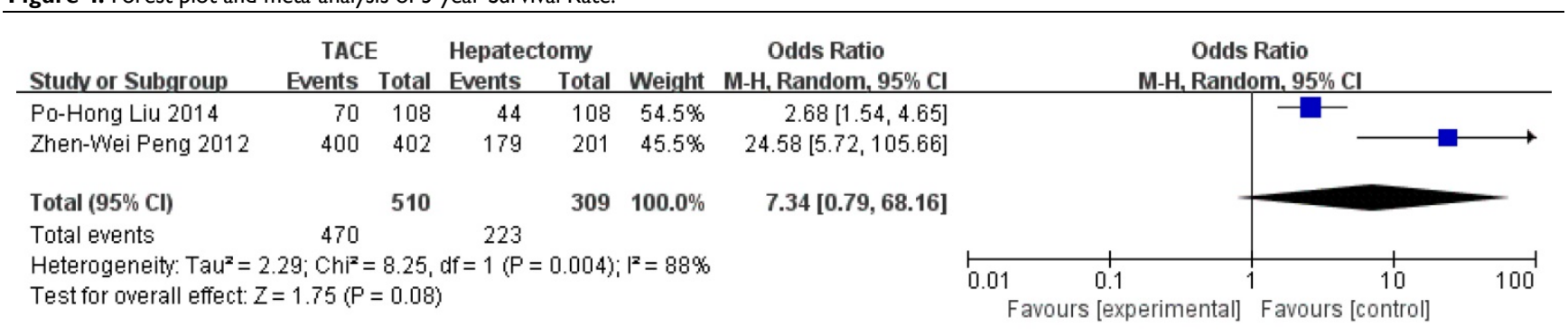

Figure 5. Forest plot and meta-analysis of 5-year Survival Rate. 


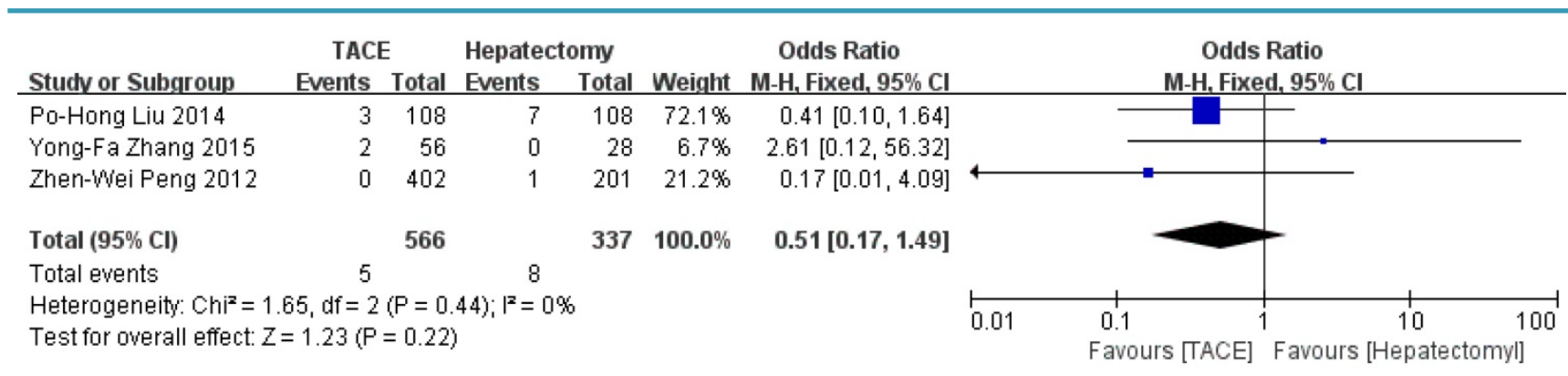

Figure 6. Forest plot and meta-analysis of peri-operative mortality.

Table 3. Results of meta-analysis comparison of TACE and hepatectomy

\begin{tabular}{|c|c|c|c|c|c|c|c|c|c|}
\hline \multirow[t]{2}{*}{ Outcome } & \multirow[t]{2}{*}{ Studies numbers } & \multirow[t]{2}{*}{ TACE } & \multirow[t]{2}{*}{ Hepatectomy } & \multirow[t]{2}{*}{$\mathrm{OR} / \mathrm{HR}$} & \multirow[t]{2}{*}{$P$ value } & \multicolumn{4}{|c|}{ Heterogeneity } \\
\hline & & & & & & $\mathrm{X}^{2}$ & df & $\mathrm{P}$ & $I^{2}$ \\
\hline Over survival & 8 & 830 & 853 & $1.61[1.23,2.10]$ & 0.0005 & 29.55 & 7 & 0.0001 & $76 \%$ \\
\hline PVTT subgroup & 6 & 754 & 790 & $1.50[1.14,1.98]$ & 0.004 & 22.99 & 6 & 0.0003 & $78 \%$ \\
\hline HVTT/IVC subgroup & 2 & 76 & 63 & $2.39[0.88,6.49]$ & 0.09 & 3.46 & 1 & 0.06 & $71 \%$ \\
\hline 1- year survival rate & 8 & 830 & 853 & $2.27[1.26,4.08]$ & 0.006 & 30.93 & 7 & $<0.0001$ & $77 \%$ \\
\hline 3- year survival rate & 7 & 768 & 580 & $3.04[2.17,4.26]$ & $<0.00001$ & 9.84 & 6 & 0.13 & $39 \%$ \\
\hline 5- year survival rate & 2 & 510 & 309 & $7.34[0.79,68.16]$ & 0.08 & 8.25 & 1 & 0.004 & $88 \%$ \\
\hline Peri-operatvie mortality & 3 & 566 & 337 & $0.51[0.17,1.49]$ & 0.22 & 1.65 & 2 & 0.44 & $0 \%$ \\
\hline \multicolumn{10}{|l|}{ Sensitivity analysis } \\
\hline Over survival & 5 & 356 & 341 & $1.74[1.45,2.10]$ & $<0.00001$ & 5.97 & 4 & 0.2 & $33 \%$ \\
\hline 1- year survival rate & 5 & 356 & 341 & $3.37[1.91,5.95]$ & $<0.00001$ & 8.01 & 4 & 0.09 & $50 \%$ \\
\hline
\end{tabular}

With the development of surgical skills, surgical instruments and peri-operative management, hepatectomy combined with thrombectomy become safe and effective. It may provide the following benefits to HCC patients with MaVI: 1) remove the thrombi and decrease the portal venous pressure which may lead to bleeding of esophageal varice; 2) reduce tumor burden; 3) improvement in quality of life; 4) increase the efficacy of post-operative treatments and prolongation in survivals [11, 23]. Recently more and more studies demonstrated that survival rates were improving for HCC patients with MaVI undergoing hepatectomy [11]. Kojima $\mathrm{H}$ et al. reported a median DFS of 8.9 months and an OS time of 27.6 months [11]. Also the types of portal vein tumor thrombosis is relevant to the survival benefit of patients with PVTT $[23,24]$. Ban D et al. reported that the 3- and 5-year survival rates in vp3 (tumor thrombus in the first branch of the portal vein) and vp4 (tumor thrombus in the portal trunk or extending to a branch on the contralateral side) group were $35.3 \%$ and $41.8 \%$, and $21.2 \%$ and $20.9 \%$ respectively [24]. Xu JF et al. also demonstrated that liver resection combined with thrombectomy for $\mathrm{HCC}$ with PVTT got better outcome in the HCC patients with PVTT involving only one branch (left/right) of portal vein compared to patients with PVTT involving the main portal vein trunk or both the left and right portal veins [23].

TACE is another choice for HCC patients with MaVI. But if main portal vein obstruction is recovered, TACE was theoretically contraindicated because of the potential risk of hepatic insufficiency resulting from ischemia after the procedure [25].Several retrospective and prospective studies had approved the efficacy and safety of TACE for these patients $[12,13,26,27]$. A prospective study enrolling 164 patients showed that TACE was safe and feasible in patients with unresectable HCC with PVTT [26]. The TACE group had significantly better survivals than the conservative group; and the 12- and 24-month OS for the TACE and conservative groups were $30.9 \%$ and $9.2 \%, 3.8 \%$ and $0 \%$, respectively; and the wide extent of thrombi correlated negatively with survival in patients undergoing TACE. The complications, characterized by nausea, vomiting, abdominal pain and fever are slight and controllable; almost all of them could be treated successfully with conservative treatment and none of TACE-related death is reported.

All studies included in meta-analysis demonstrated significantly better overall survival for patients treated with hepatectomy than those treated with TACE as initial treatment. But it had to be comment that those patients who received hepatecomy were highly selected. When a better survival can be expected, the aggressive treatment is more likely to be accepted. In some retrospective studies, patients undergoing hepatectomy were younger, in better performance status, better liver function and less tumor nodules [17]. These differences tend to a better survival in hepatectomy group. To minimize the selection bias, some studies were designed as case-matched controls. In order to 
reach a consistent and comparable pretreatment baseline characteristics of patients, the factors probably affect the survival were matched. However these case-matched controls also showed better survivals in hepatectomy group. Peng ZW et al. reported significantly better 1 -year $(42.0 \%$ vs $37.8 \%)$, 3 -year $(14.1 \%$ vs $7.3 \%)$ and 5 -year $(11.1 \%$ vs $0.5 \%)$ OS in hepatectomy group [21]. They further conduct a subgroup analysis in different types of MaVI. The results demonstrated that the overall survivals for the hepatectomy group were better than the TACE group for type I PVTT (tumor thrombi involving the segmental branches of the portal vein or above), type II PVTT (tumor thrombi extending to involve the right/left portal vein), but not for type III PVTT (the main portal vein), and type IV PVTT (the superior mesenteric vein). When it comes to the complication after treatment, hepatectomy group and TACE group couldn't reach a significant differ.

It must be commented that all 8 studies included in our meta-analysis were from Asia, 1 from Korean and 7 from China, none from Europe or American. As we all know, for patients with MaVI, namely BCLC C stage or T4 in TNM stage, sorafenib is the only recommended option in the West, as a result, few experts would accept hepatectomy or TACE as treatment option for those patients. On the contrary, both treatments are widely accepted in Asia, which might contribute to the different in etiology and biological behavior between the East and the West HCC. HCV infection is a more important factor than HBV in the development of HCC in western countries, and it is also believed that there are lots of clinical differences between HBV- and HCV-related HCC. In most patients with HCV-related HCC, the tumors are more likely to be solitary, smaller sized and encapsulated whereas HBV-related HCC are more commonly infiltrative, multi-nodular, and portal vein invasion. On the other side, a higher proportion of HCV-related HCC than HBV-related HCC has advanced liver histology and has a higher Child Pugh's score, which preclude the patients from aggressive treatments such as hepatectomy and TACE. However, in the past decades, some surgeons from the Western countries reported their experiences in hepatectmy for HCC patients with MaVI, but the results were controversial. It highlights the importance to investigate this issue in the future.

The limitations of this meta-analysis must be acknowledged. Firstly, all of the included studies are retrospective studies. No high-quality prospective randomized clinical trial in such subject can be found on the databases chosen. Thus, the results of the studies will be influenced by selection bias. Second, the follow-up periods of these including studies were generally short, the long-term survival benefit between two groups remain to be proved. Third, the heterogeneity was generally significant in our meta-analysis. Bias coming from different levels of expertise, different chemo agents in different centers or different baseline characteristics and intervention protocols must be further analyzed.

This meta-analysis indicated that hepatectomy might be a better treatment option for resectable HCC with MaVI, comparing with TACE. It associated with a significant survival benefit and did not increase peri-operative mortality. Being lack of high-quality RCT, more well-designed multi-centre random clinical trials are needed to prove our outcomes.

\section{Acknowledgement}

This study was funded by the National Natural Science Foundation of China (NSFC81572387 and 81602143), the "5010 program" of Sun Yat-Sen University (2013009), and the Scientific Project of Guangdong Province (2017A020215034).

\section{Ethics approval and consent to participate}

All procedures performed in studies involving human participants were in accordance with the ethical standards of the institutional and/or national research committee and with the 1964 Helsinki declaration and its later amendments or comparable ethical standards.

\section{Consent for publication} authors.

Publication consent was obtained from all

\section{Competing Interests}

The authors have declared that no competing interest exists.

\section{References}

1 Torre LA, Bray F, Siegel RL, Ferlay J, Lortet-Tieulent J, Jemal A. Global cancer statistics, 2012. CA Cancer J Clin 2015; 65: 87-108.

2 Minagawa M, Makuuchi M. Treatment of hepatocellular carcinoma accompanied by portal vein tumor thrombus. World journal of gastroenterology 2006; 12: 7561-7567.

3 Nishikawa $H$, Kita R, Kimura T, Ohara $Y$, Takeda $H$, Sakamoto A et al. Transcatheter arterial chemoembolization for intermediate-stage hepatocellular carcinoma: clinical outcome and safety in elderly patients. J Cancer 2014; 5: 590-597.

4 Llovet JM, Bustamante J, Castells A, Vilana R, Ayuso Mdel C, Sala M et al. Natural history of untreated nonsurgical hepatocellular carcinoma: rationale for the design and evaluation of therapeutic trials. Hepatology 1999; 29: 62-67.

5 Villa E, Moles A, Ferretti I, Buttafoco P, Grottola A, Del Buono M et al. Natural history of inoperable hepatocellular carcinoma: estrogen receptors' status in the tumor is the strongest prognostic factor for survival. Hepatology 2000; 32: 233-238.

6 Ye JZ, Zhang YQ, Ye HH, Bai T, Ma L, Xiang BD et al. Appropriate treatment strategies improve survival of hepatocellular carcinoma patients with portal vein tumor thrombus. World journal of gastroenterology 2014; 20: 17141-17147.

7 Llovet JM, Ricci S, Mazzaferro V, Hilgard P, Gane E, Blanc JF et al. Sorafenib in advanced hepatocellular carcinoma. N Engl J Med 2008; 359: 378-390.

8 Cheng AL, Kang YK, Chen Z, Tsao CJ, Qin S, Kim JS et al. Efficacy and safety of sorafenib in patients in the Asia-Pacific region with advanced hepatocellular 
carcinoma: a phase III randomised, double-blind, placebo-controlled trial. Lancet Oncol 2009; 10: 25-34

9 Nishikawa H, Takeda H, Tsuchiya K, Joko K, Ogawa C, Taniguchi $\mathrm{H}$ et al. Sorafenib Therapy for BCLC Stage B/C Hepatocellular Carcinoma; Clinical Outcome and Safety in Aged Patients: A Multicenter Study in Japan. J Cancer 2014; 5: 499-509.

10 Kokudo T, Hasegawa K, Matsuyama Y, Takayama T, Izumi N, Kadoya Met al. Survival benefit of liver resection for hepatocellular carcinoma associated with portal vein invasion. Journal of hepatology 2016; 65: 938-943.

11 Kojima H, Hatano E, Taura K, Seo S, Yasuchika K, Uemoto S. Hepatic Resection for Hepatocellular Carcinoma with Tumor Thrombus in the Major Portal Vein. Digestive surgery 2015; 32: 413-420.

12 Liu L, Zhang C, Zhao Y, Qi X, Chen H, Bai W et al. Transarterial chemoembolization for the treatment of advanced hepatocellular carcinoma with portal vein tumor thrombosis: prognostic factors in a single-center study of 188 patients. BioMed research international 2014; 2014: 194278.

13 Niu ZJ, Ma YL, Kang P, Ou SO, Meng ZB, Li ZK et al. Transarterial chemoembolization compared with conservative treatment for advanced hepatocellular carcinoma with portal vein tumor thrombus: using a new classification. Medical oncology (Northwood, London, England) 2012; 29: 2992-2997.

14 Tierney JF, Stewart LA, Ghersi D, Burdett S, Sydes MR. Practical methods for incorporating summary time-to-event data into meta-analysis. Trials 2007; 8: 16.

15 Zhang Y-F, Wei W, Guo Z-X, Wang J-H, Shi M, Guo R-P. Hepatic resection versus transcatheter arterial chemoembolization for the treatment of hepatocellular carcinoma with hepatic vein tumor thrombus. Japanese journal of clinical oncology 2015; 45: 837-843.

16 Wang $\mathrm{Z}, \mathrm{Hu} \mathrm{K}$, Tao Y. Surgical treatment strategy for primary liver cancer with portal vein tumor thrombus: a report of 335 cases. Chinese Journal of General Surgery 2015; 24: 928-932.

17 Liu PH, Lee YH, Hsia CY, Hsu CY, Huang YH, Chiou YY et al. Surgical resection versus transarterial chemoembolization for hepatocellular carcinoma with portal vein tumor thrombosis: a propensity score analysis. Annals of surgical oncology 2014; 21: 1825-1833.

18 Lee Y-H, Hsu C-Y, Huang Y-H, Hsia C-Y, Chiou Y-Y, Su C-W et al. Vascular Invasion in Hepatocellular Carcinoma Prevalence, Determinants and Prognostic Impact. Journal of Clinical Gastroenterology 2014; 48: 734-741.

19 Wang Y, Yuan L, Ge RL, Sun Y, Wei G. Survival benefit of surgical treatment for hepatocellular carcinoma with inferior vena cava/right atrium tumor thrombus: results of a retrospective cohort study. Annals of surgical oncology 2013; 20: 914-922.

20 Ma L, Ye J, Xiang B, Wu F, Zhao Y, Li L. Impact of treatment strategies on patients with hepatocellular carcinoma of less than $10 \mathrm{~cm}$ but with portal vein tumor thrombus. Chinese Journal of Hepatobiliary Surgery 2013; 19: 165-170.

21 Peng ZW, Guo RP, Zhang YJ, Lin XJ, Chen MS, Lau WY. Hepatic resection versus transcatheter arterial chemoembolization for the treatment of hepatocellular carcinoma with portal vein tumor thrombus. Cancer 2012; 118: 4725-4736.

22 Zhou Q, Wang Y, Zhou X, Peng B, Yang J, Liang L et al. Prognostic Analysis for Treatment Modalities in Hepatocellular Carcinomas with Portal Vein Tumor Thrombi. Asian Pacific Journal of Cancer Prevention 2011; 12: 2847-2850.

$23 \mathrm{Xu} \mathrm{JF}$, Liu XY, Wang S, Wen HX. Surgical treatment for hepatocellular carcinoma with portal vein tumor thrombus: a novel classification. World journal of surgical oncology 2015; 13: 86 .

24 Ban D, Shimada K, Yamamoto Y, Nara S, Esaki M, Sakamoto Y et al. Efficacy of a hepatectomy and a tumor thrombectomy for hepatocellular carcinoma with tumor thrombus extending to the main portal vein. Journal of gastrointestinal surgery : official journal of the Society for Surgery of the Alimentary Tract 2009; 13: 1921-1928.

25 Chung JW, Park JH, Han JK, Choi BI, Han MC, Lee HS et al. Hepatic tumors: predisposing factors for complications of transcatheter oily chemoembolization. Radiology 1996; 198: 33-40.

26 Luo J, Guo RP, Lai EC, Zhang YJ, Lau WY, Chen MS et al. Transarterial chemoembolization for unresectable hepatocellular carcinoma with portal vein tumor thrombosis: a prospective comparative study. Annals of surgical oncology 2011; 18: 413-420.

27 Tawada A, Chiba T, Ooka Y, Kanogawa N, Motoyama T, Saito T et al. Efficacy of transarterial chemoembolization targeting portal vein tumor thrombus in patients with hepatocellular carcinoma. Anticancer research 2014; 34: $4231-4237$. 